University of New Hampshire

University of New Hampshire Scholars' Repository

Physics Scholarship

Physics

8-2014

\title{
Generation of unusually low frequency plasmaspheric hiss
}

\author{
Lunjin Chen \\ University of California - Los Angeles \\ R. M. Thorne \\ University of California - Los Angeles \\ J. Bortnik \\ University of California - Los Angeles \\ Wen Li \\ University of California - Los Angeles \\ Richard B. Horne \\ British Antarctic Survey
}

See next page for additional authors

Follow this and additional works at: https://scholars.unh.edu/physics_facpub

Part of the Physics Commons

\section{Recommended Citation}

Chen, L., et al. (2014), Generation of unusually low frequency plasmaspheric hiss, Geophys. Res. Lett., 41, 5702-5709, doi:10.1002/2014GL060628

This Article is brought to you for free and open access by the Physics at University of New Hampshire Scholars' Repository. It has been accepted for inclusion in Physics Scholarship by an authorized administrator of University of New Hampshire Scholars' Repository. For more information, please contact Scholarly.Communication@unh.edu. 


\section{Authors}

Lunjin Chen, R. M. Thorne, J. Bortnik, Wen Li, Richard B. Horne, Geoffrey Reeves, C A. Kletzing, W. S. Kurth, G. B. Hospodarsky, Harlan E. Spence, J. B. Blake, and Joseph F. Fennell 


\section{Geophysical Research Letters}

\author{
RESEARCH LETTER \\ 10.1002/2014GL060628 \\ Key Points: \\ - Repeated amplification due to \\ cyclic path leads to unusually low \\ frequency hiss \\ - The cyclic raypaths of waves at several \\ tens $\mathrm{Hz}$ are relatively stable \\ - The newly injected electrons \\ inside the plasmasphere are only \\ weakly unstable
}

Correspondence to:

L. Chen,

lunjin.chen@gmail.com

\section{Citation:}

Chen, L., et al. (2014), Generation of unusually low frequency plasmaspheric hiss, Geophys. Res. Lett., 41, 5702-5709, doi:10.1002/2014GL060628.

\section{Received 22 MAY 2014} Accepted 25 JUN 2014 Accepted article online 27 JUN 2014 Published online 20 AUG 2014

\section{Generation of unusually low frequency plasmaspheric hiss}

\author{
Lunjin Chen ${ }^{1}$, Richard M. Thorne ${ }^{2}$, Jacob Bortnik², Wen Li ${ }^{2}$, Richard B. Horne ${ }^{3}$, G. D. Reeves ${ }^{4}$, \\ C. A. Kletzing ${ }^{5}$, W. S. Kurth ${ }^{5}$, G. B. Hospodarsky ${ }^{5}$, H. E. Spence ${ }^{6}$, J. B. Blake ${ }^{7}$, and J. F. Fennell ${ }^{7}$
}

${ }^{1}$ Department of Physics, University of Texas at Dallas, Richardson, Texas, USA, ${ }^{2}$ Department of Atmospheric and Oceanic Sciences, University of California, Los Angeles, California, USA, ${ }^{3}$ British Antarctic Survey, Natural Environment Research Council, Cambridge, UK, ${ }^{4}$ Space Science and Applications Group, Los Alamos National Laboratory, Los Alamos, New Mexico, USA, ${ }^{5}$ Department of Physics and Astronomy, University of lowa, lowa, lowa, USA, ${ }^{6}$ Institute for the Study of Earth, Oceans, and Space, University of New Hampshire, Durham, New Hampshire, USA, ${ }^{7}$ The Aerospace Corporation, Los Angeles, California, USA

Abstract It has been reported from Van Allen Probe observations that plasmaspheric hiss intensification in the outer plasmasphere, associated with a substorm injection on 30 September 2012, occurred with a peak frequency near $100 \mathrm{~Hz}$, well below the typical plasmaspheric hiss frequency range, extending down to $\sim 20 \mathrm{~Hz}$. We examine this event of unusually low frequency plasmaspheric hiss to understand its generation mechanism. Quantitative analysis is performed by simulating wave raypaths via the HOTRAY ray tracing code with measured plasma density and calculating raypath-integrated wave gain evaluated using the measured energetic electron distribution. We demonstrate that the growth rate due to substorm-injected electrons is positive but rather weak, leading to small wave gain $(\sim 10 \mathrm{~dB})$ during a single equatorial crossing. Propagation characteristics aided by the sharp density gradient associated with the plasmapause, however, can enable these low-frequency waves to undergo cyclic raypaths, which return to the unstable region leading to repeated amplification to yield sufficient net wave gain ( $>40 \mathrm{~dB}$ ) to allow waves to grow from the thermal noise.

\section{Introduction}

Plasmaspheric hiss is an incoherent, broadband, electromagnetic whistler mode emission which is preferentially observed inside the high-density plasmasphere [Russell et al., 1969; Thorne et al., 1973] and storm-time plasmaspheric plume [Summers et al., 2008]. Plasmaspheric hiss has a typical frequency range from $\sim 100 \mathrm{~Hz}$ to $\sim 2 \mathrm{kHz}$ [Thorne et al., 1973; Meredith et al., 2004], with most power between $\sim 200 \mathrm{~Hz}$ and $1 \mathrm{kHz}$ [Smith et al., 1974; Hayakawa and Sazhin, 1992]. Since its discovery in the late 1960s, a number of theories have been proposed to explain the origin of this emission. Electron cyclotron instability inside the plasmasphere occasionally provides sufficient wave gain [Solomon et al., 1988] but most of the time is too weak to account for the observed emission [Huang et al., 1983; Church and Thorne, 1983]. Lightning-generated whistlers have been proposed as the embryonic source of plasmaspheric hiss [Draganov et al., 1992; Sonwalkar and Inan, 1989; Green et al., 2005], but later studies [Meredith et al., 2006] found that this was only linked to plasmaspheric hiss above $2 \mathrm{kHz}$, which is not in the main frequency range of plasmaspheric hiss. Recent ray tracing simulations [Bortnik et al., 2008] and simultaneous observations of chorus and hiss emissions [Bortnik et al., 2009] have suggested that plasmaspheric hiss originates from chorus emissions generated outside the plasmasphere, which is also consistent with statistical findings from the Polar satellite [Tsurutani et al., 2012]. This mechanism can account for the plasmaspheric hiss in the typical frequency range $200-2000 \mathrm{~Hz}$ as well as many other characteristic features [Chen et al., 2012a, 2012b], including the spatial distribution and intensity of the observed plasmaspheric hiss.

Li et al. [2013] recently reported an intensification of the plasmaspheric hiss emission observed from the twin Van Allen Probes [Mauk et al., 2012] at frequencies down to $\sim 20 \mathrm{~Hz}$ and with the frequency of peak power just below $100 \mathrm{~Hz}$, much lower than typical hiss wave frequencies. Figure 1a shows the magnetic field spectral density during this event observed by the Electric and Magnetic Field Instrument Suite and Integrated Science (EMFISIS) wave instrument [Kletzing et al., 2013] during the outbound pass of Van Allen Probe A from 14:00 UT to 19:30 UT, on 30 September 2012. The spectral intensity is plotted against $L$ shell and so is the plasma density inferred from the spacecraft potential (blue dots), which is consistent with upper hybrid resonance line measurement from the EMFISIS. Plasmaspheric hiss waves at such low 


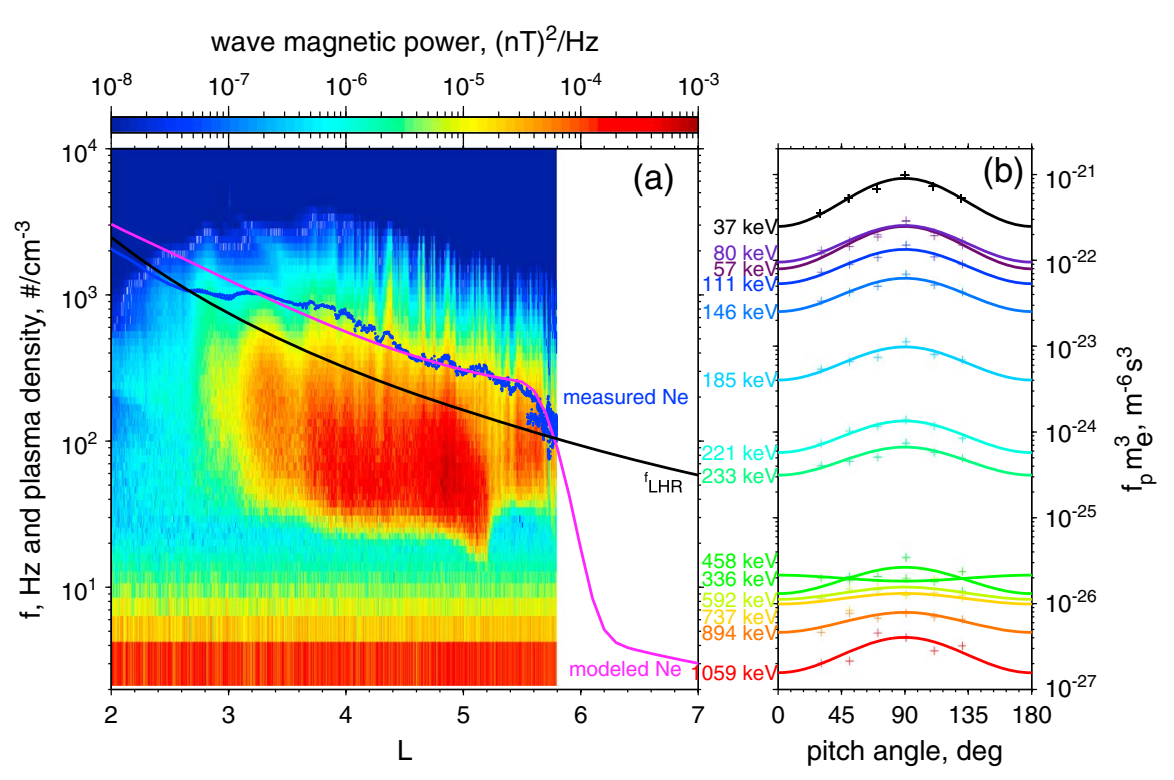

Figure 1. (a) The observed magnetic field spectral density in the waveform receiver of the EMFISIS wave instrument on Van Allen Probe A over the time period 14:00-19:30 UT of 30 September 2012, organized as a function of $L$ shell and frequency. The blue dots represents the plasma density inferred from the spacecraft potential, and the magenta line denotes the modeled equatorial profile used for the ray tracing simulation. The black line denotes the local lower hybrid resonance frequency $f_{\mathrm{LHR}}$ at the equator. (b) The measured (pluses) and modeled (solid lines) electron relativistic momentum phase space density $f_{p}$ at $L=5$ as a function of pitch angle over energy channels of the MagEIS instrument from $37 \mathrm{keV}$ to $1059 \mathrm{keV}$. The electron mass is denoted by $m_{e}$ in the vertical label.

frequency are unlikely linked to chorus waves [Chen et al., 2012a], because the chorus mechanism would require chorus waves generated beyond $L \sim 9$, which would be substantially attenuated by Landau damping before reaching the plasmasphere (due to long raypath). The intensification of the unusually low frequency plasmaspheric hiss occurred in the outer plasmasphere and is strongly correlated with substorm-injected energetic electrons [Li et al., 2013], suggesting that the plasmaspheric hiss is likely amplified locally due to the free energy in the injected electron population. Although previous observations [e.g., Tsurutani et al., 1975] show weak hiss emission extending below $200 \mathrm{~Hz}$, the observed emission in our study is unusual not only due to its spectral intensity peak below $200 \mathrm{~Hz}$ but also due to the rare condition associated, i.e., the injection of energetic electrons into the dawnside plasmasphere during the substorm. Figure $1 \mathrm{~b}$ shows the electron pitch angle distribution of electron momentum phase space density from the Magnetic Electron Ion Spectrometer (MagEIS) instrument [Blake et al., 2013] of the Energetic Particle Composition and Thermal Plasma Suite [Spence et al., 2013] at energy channels from $37 \mathrm{keV}$ to $1059 \mathrm{keV}$ at $L \sim 5$, corresponding to the region where the unusually low frequency plasmaspheric hiss emission was observed. The injected electrons clearly show pitch angle anisotropy, and that provides a positive growth rate for the whistler mode emissions. However, whether the instability due to these enhanced electron fluxes provides sufficient wave gain to produce the observed unusually low frequency plasmaspheric hiss emission needs to be carefully evaluated. By using the Van Allen Probes measurements of the actual plasma and wave parameters, we address this question to understand the physical mechanism responsible for intensification of this low-frequency hiss emission. We use ray tracing to simulate raypaths of plasmaspheric hiss waves and the observed energetic electron distribution (Figure $1 b$ ) to calculate the path-integrated wave gain. The simulation results are presented in section 2, followed by conclusions and a discussion in section 3 .

\section{Model and Results}

To model the propagation characteristics of plasmaspheric hiss waves, we use the HOTRAY ray tracing code [Horne, 1989], where a magnetic dipole field is adopted and a diffusive equilibrium plasma density model [Bortnik et al., 2011] is adopted with an equatorial density profile (magenta line of Figure 1a) fitted to the measured plasma density (blue dots of Figure 1a). The modeled plasmapause is located at $L \sim 5.8$ for the inner edge and at $L \sim 6.3$ for the outer edge. Figure 2a shows a cycle of a raypath (over $\sim 24 \mathrm{~s}$ ) in the 2-D meridian plane for a whistler mode wave with a frequency of $f=80 \mathrm{~Hz}$, launched at the equator at 

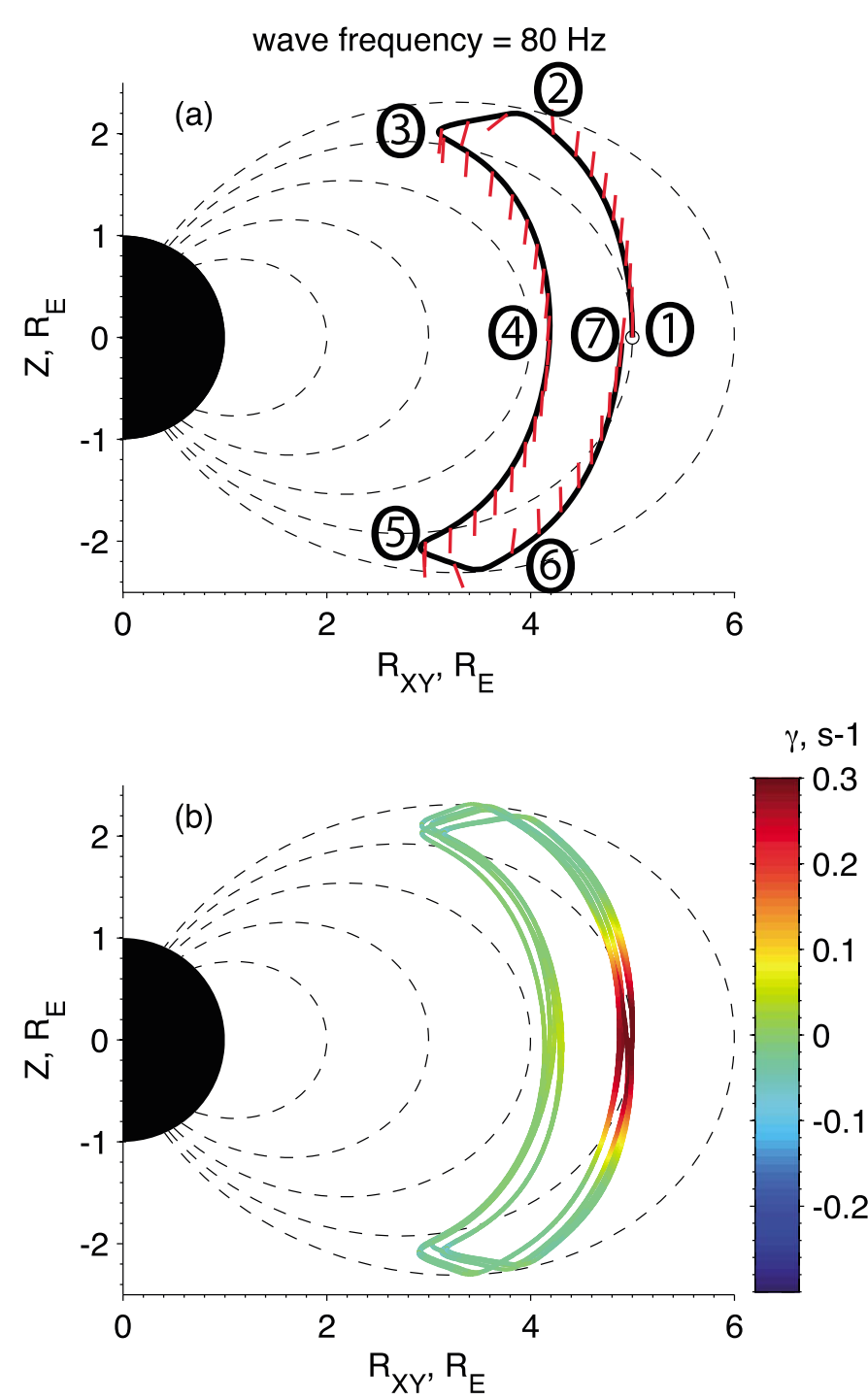

0.3

0.2

0.1

0

$-0.1$

$-0.2$
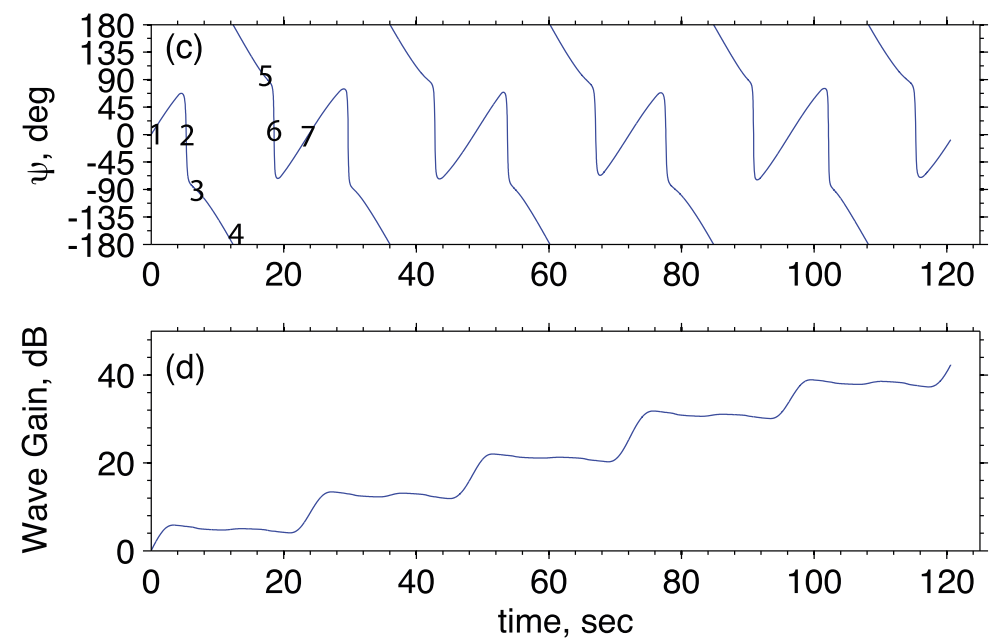

Figure 2. (a) The raypath of an $80 \mathrm{~Hz}$ wave launched at $L=5$ during the first cycle. The short red segments represent the instantaneous wave normal directions along the raypath. (b) The raypath of the wave over the first five cycles with color-coded local temporal growth rate along the raypath. (c) Wave normal angle $\psi$ and (d) path-integrated wave gain as a function of propagation time. The dashed lines in Figures $2 \mathrm{a}$ and $2 \mathrm{~b}$ represent dipole magnetic field of $L=2,3,4,5$, and 6 . Seven characteristic points during the first cycle are marked by numbers in Figures $2 \mathrm{a}$ and $2 \mathrm{c}$. 
$L_{0}=5$ with initial wave normal angle $\psi_{0}=0^{\circ}$. A cycle is defined as a ray passing through the equator from the Southern Hemisphere to the Northern Hemisphere. The wave normal angle $\psi$ is defined so that $\psi$ has a range of $\left(-180^{\circ}, 180^{\circ}\right)$ with positive (or negative) values indicating direction toward larger (or smaller) L. During the first cycle, this ray undergoes the following characteristic steps: (1) propagating along the field-aligned direction $\left(\psi=0^{\circ}\right)$ at the equator toward the Northern Hemisphere; (2) refracting back from the plasmapause due to the steep plasma density gradient across magnetic field lines, with $\psi=0^{\circ}$ during the bounce; (3) reflecting at high latitude $\left(\psi=-90^{\circ}\right)$ at the Northern Hemisphere and then propagating toward the south; (4) passing through the equator from the north to the south at $L$ less than $L_{0}$ with antiparallel propagation $\psi \sim-180^{\circ}$; (5) reflecting $\left(\psi=90^{\circ}\right)$ at the Southern Hemisphere and then propagating toward the north; (6) refracting back from the plasmapause at the Southern Hemisphere with $\psi=0^{\circ}$ during the bounce; and (7) reaching the equator at the end of the cycle, with near field-aligned propagation $\left(\psi \sim 0^{\circ}\right)$ at $L$ near $L_{0}(\sim 5)$, which is almost the same configuration as the initial one at $t=0$. By returning nearly to the initial launching configuration, the ray will follow a similar raypath for subsequent cycles. The raypath and wave normal angle $\psi$ over the first five cycles are shown in Figures $2 \mathrm{~b}$ and $2 \mathrm{c}$ respectively. This type of raypath has been described as a cyclic raypath earlier by Thorne et al. [1979]. It should be noted that the propagation characteristics of this low-frequency wave during reflection are somewhat different from previously mentioned "magnetospheric reflection" or "MR" whistlers [Kimura, 1966]. Magnetospherically reflected waves rotate their wave normal direction rapidly through $90^{\circ}$ when the waves propagate from the region where the wave frequency is greater than the local lower hybrid resonance frequency $\left(f_{L H R}\right)$ and thus the refractive index surface is open, to a region where the wave frequency is just less than the local $f_{L H R}$ and thus the refractive index surface is closed. For this ray, the wave frequency $(80 \mathrm{~Hz})$ is always below the local $f_{\text {LHR }}$ inside the plasmasphere, which is always greater than $\sim 100 \mathrm{~Hz}$ for $L<6$ (shown by the black line of Figure 1a). As a consequence, the refractive index surface is always closed, and the raypath during the reflection appears less sharp, especially so for even lower frequencies.

The temporal growth rate can also be evaluated along the raypath using the modeled electron distribution as shown in Figure $1 \mathrm{~b}$, which is obtained by fitting the observed electron distribution near $L \sim 5$ with a functional form $f_{p}=A \exp \left(B \sin ^{2} \alpha\right)$, where $f_{p}$ is the momentum phase space density, $\alpha$ is the electron pitch angle, and $A$ and $B$ are the two fitting parameters. We assume the electron momentum distributions are the same spatially throughout the plasmasphere and constant, which is not a critical assumption because, as is shown later, most of the growth takes place near $L \sim 5$ and also because the time scale of quasilinear relaxation is much longer than duration time scale of substorm injection ( $30 \mathrm{~min})$ and the ray tracing simulation time. Figure $2 \mathrm{~b}$ shows the linear temporal growth rate based on the electron distribution ( $37 \mathrm{keV}-1059 \mathrm{keV}$ ) and including resonances with $n$ from -5 to +5 (i.e., $n=-1$ for primary cyclotron resonance and $n=0$ for Landau resonance), according to the relativistic growth rate formula developed by Xiao [2001]. Because the electron distribution drops by $>1$ order of magnitude between the $233 \mathrm{keV}$ and the $336 \mathrm{keV}$ channels, the growth rate is controlled by electrons with energy $\leq 233 \mathrm{keV}$. Clearly, the maximum growth rate occurs for the equatorial crossings that are closer to the plasmapause (Figure $2 \mathrm{~b}$ ), due to $\psi \sim 0^{\circ}$ and larger electron plasma frequency to gyrofrequency ratios. Accordingly, the increase of the path-integrated wave gain (Figure $2 \mathrm{~d}$ ) is mostly attributed to the outer equatorial crossings and is relatively small for the lower $L$ equatorial crossings. The net wave gain over a cycle is rather small ( $8 \mathrm{~dB})$, suggesting that the free energy due to the observed anisotropic electron distribution is not sufficient to provide amplification from the background noise level to the observed intensity through a single equatorial passage. However, the repeated amplification over multiple cycles (Figure $2 \mathrm{~d}$ ) can enhance the wave gain continuously ( $>40 \mathrm{~dB}$ over five cycles or $\sim 120 \mathrm{~s}$ ).

Similar ray tracing simulations were run for a range of wave frequencies from $20 \mathrm{~Hz}$ to $500 \mathrm{~Hz}$ with a frequency spacing of $10 \mathrm{~Hz}$ to represent the observed frequency range of the plasmaspheric hiss intensification event near $L=5$. All these waves show a cyclic type of raypaths inside the plasmasphere and experience repeated amplification on each cycle. Path-integrated wave gain at the end of each cycle for the first eight cycles and the time to complete the first cycle are plotted against wave frequency in Figures $3 a$ and $3 \mathrm{~b}$ respectively. The wave gain peaks near $200 \mathrm{~Hz}$ with a gain of $\sim 17 \mathrm{~dB}$ per cycle $(\sim 16 \mathrm{~s})$, where the repeated amplification leads to more than $130 \mathrm{~dB}$ over eight cycles (over a time period of $\sim 2 \mathrm{~min}$ ). The peak frequency of $\sim 200 \mathrm{~Hz}$ is consistent with the frequency of peak cyclotron growth rate due to the observed electron flux (Figure 1b). There is positive growth below $100 \mathrm{~Hz}$ down to $40 \mathrm{~Hz}$, where the wave gain above $60 \mathrm{~Hz}$ exceeds $40 \mathrm{~dB}$ within eight cycles and the increasing rate of wave gain for lower frequencies 

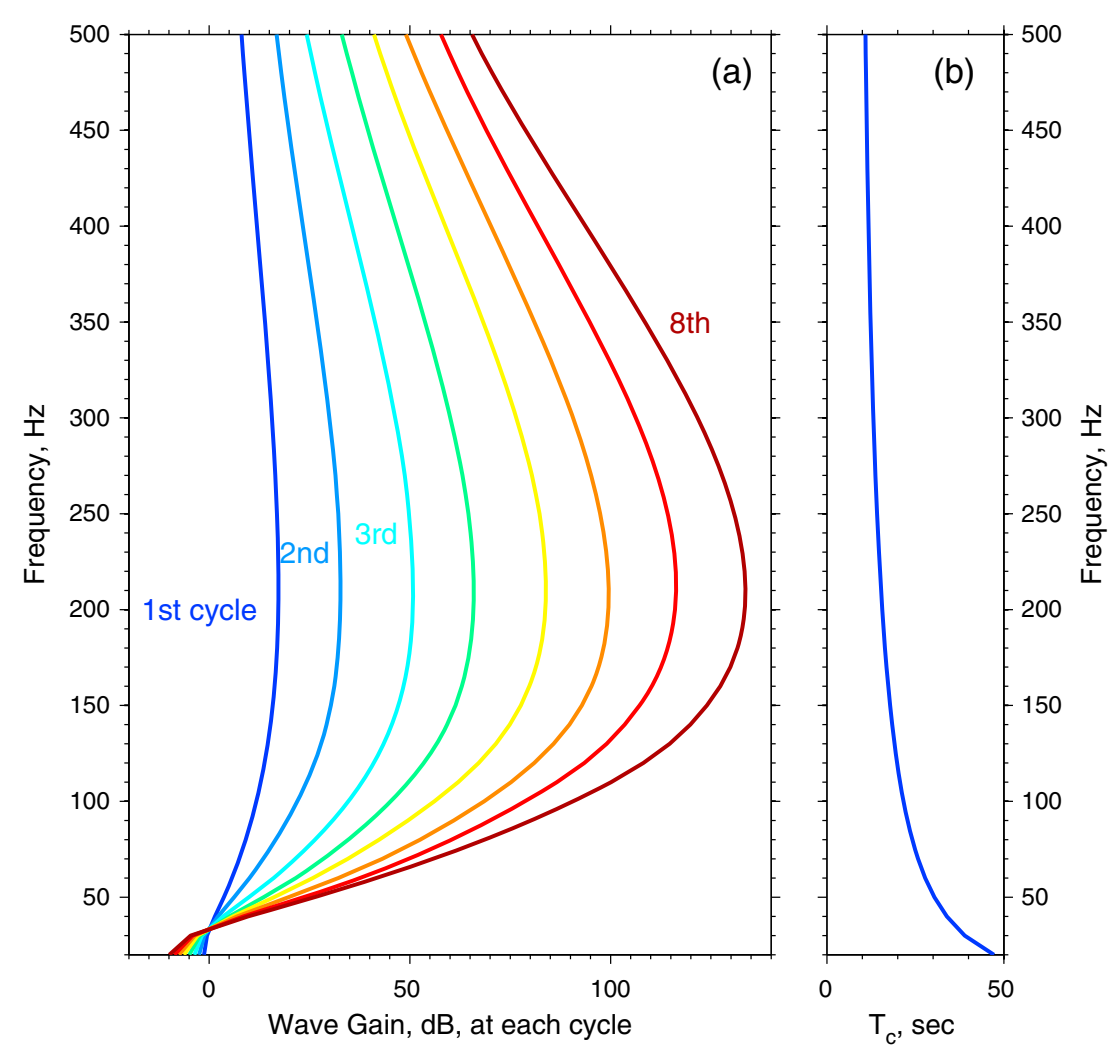

Figure 3. (a) The path-integrated wave gain as a function of wave frequency at the end of each cycle for the first eight cycles. (b) The propagation time to complete the first cycle as a function of wave frequency. A cycle is defined when a ray crosses the equator from the south to the north.

$(40-50 \mathrm{~Hz})$ is slower due to the lower wave gain per cycle and the longer time to complete a cycle. Therefore, it will take a longer time to attain a sufficient wave gain, say $40 \mathrm{~dB}$, for waves with lower frequencies. The ray with frequency $40 \mathrm{~Hz}$ takes $\sim 4$ min (eight cycles) to reach a wave gain of just below $10 \mathrm{~dB}$. Waves with even lower frequencies $<30 \mathrm{~Hz}$ experience a net damping instead of net growth because the cyclotron growth is so weak that Landau damping is dominant over the growth. One should be cautious in comparing the frequency profile of wave gain with wave frequency spectral intensity. The former represents the amplification level from the background noise level and can be translated to the latter only when the background noise level, which generally depends on frequency, is known. The wave spectral intensity peaks near $100 \mathrm{~Hz}$, while the wave gain peaks at higher frequency near $200 \mathrm{~Hz}$, probably because the background noise level becomes lower as frequency increases.

To account for the intensification of the observed plasmaspheric hiss near the lower frequency limit (several tens $\mathrm{Hz}$ ), these waves are required to experience cyclic raypaths that are stable over a relatively long time. We run the ray tracing simulation of $40 \mathrm{~Hz}$ waves launched at $L=5$ and with varying $\psi_{0}$ for $15 \mathrm{~min}$, which is within the time scale of substorm electron injection. Ray tracing terminates when one of the following occurs: when the propagation time exceeds $15 \mathrm{~min}$, or when a ray propagates beyond $L>8$ (i.e., no longer trapped by the plasmapause), or when the small-wavelength approximation fails, or when the raypath reaches the topside ionosphere. The raypaths with $\psi_{0}=0^{\circ}, \pm 20^{\circ}$, and $\pm 30^{\circ}$ are shown in Figure $4 \mathrm{a}$, and the number of cycles and the path-integrated wave gain experienced are plotted against $\psi_{0}$ in Figures $4 \mathrm{~b}$ and $4 \mathrm{c}$ respectively. The raypaths of $\left|\psi_{0}\right| \leq 20^{\circ}$ are cyclic (Figures $4 \mathrm{a}$ and $4 \mathrm{~b}$ ), and these cyclic raypaths are rather stable for the entire $15 \mathrm{~min}$ and over a broad range of $\psi_{0}$ not just for $\psi_{0}=0$. These cyclic waves experience 28 cycles over $15 \mathrm{~min}$ (Figure $4 \mathrm{~b}$ ) and therefore obtain a large cumulative path-integrated gain of 30-50 dB, depending on $\psi_{0}$. For those cyclic raypaths, the wave gain increases with $\left|\psi_{0}\right|$ (Figure $4 \mathrm{c}$ ), having a minimum wave gain for initially parallel propagating waves $\left(\psi_{0}=0\right)$. The dependence of wave gain on $\psi_{0}$ is caused by the spatial dependence of the amplification. The raypath for $\psi_{0}=0$ is almost identical during 

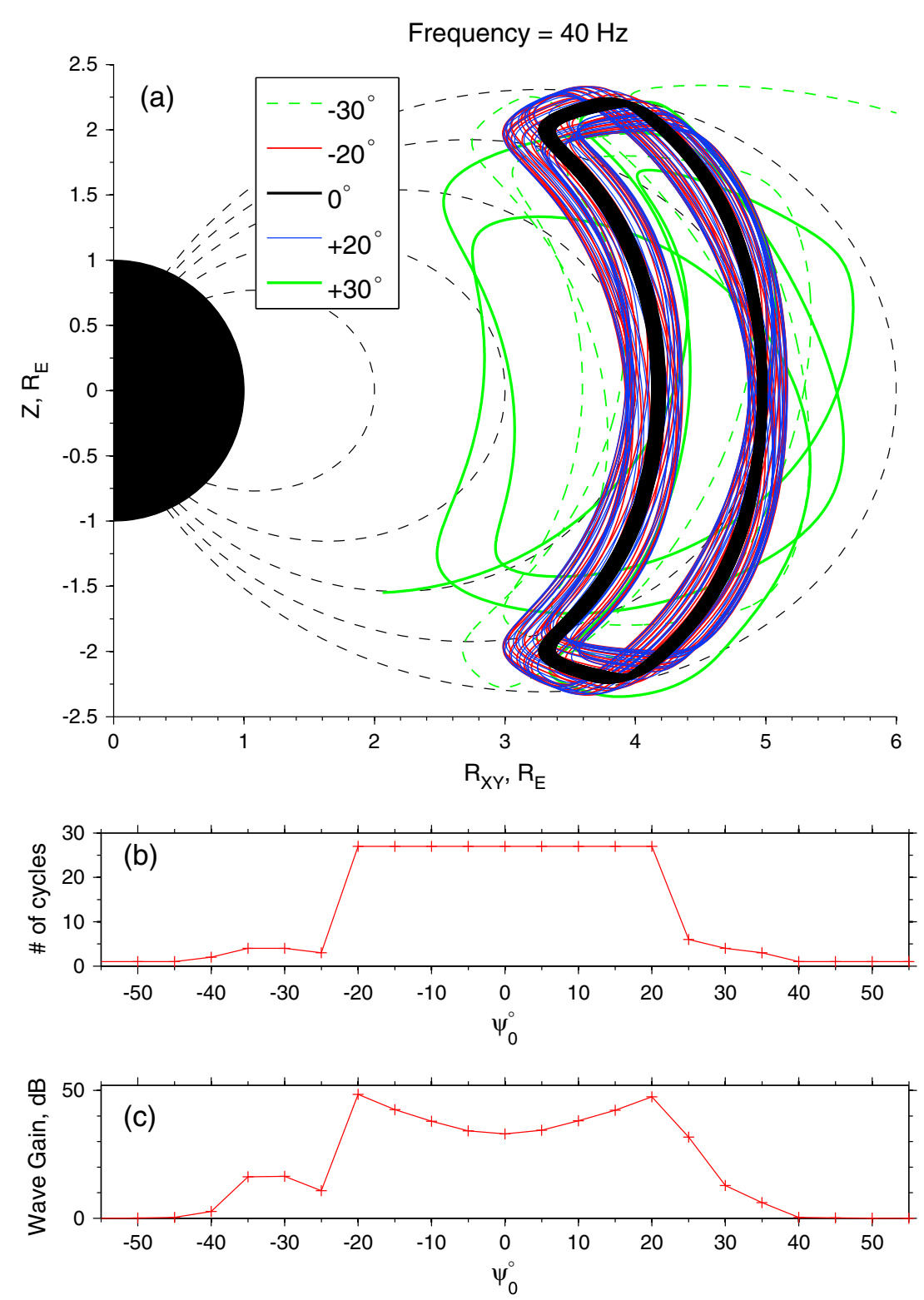

Figure 4. (a) Raypaths of $40 \mathrm{~Hz}$ waves launched at $L=5$ with varying initial wave normal angles $\psi_{0}=0^{\circ}, \pm 20^{\circ}$, and $\pm 30^{\circ}$. (b) The number of cycles experienced and (c) maximum path-integrated wave gain as a function of $\psi_{0}$.

each cycle (Figure 4a) while the raypaths for $\psi_{0}= \pm 20^{\circ}$ spread over a broader spatial region, including the spreading of outer equatorial crossings where the most amplification is expected. Outward extension of the outer equatorial crossings enhances wave amplification due to the larger electron plasma-to-gyrofrequency ratio, despite more oblique propagation during the outer equatorial crossings for $\psi_{0}= \pm 20^{\circ}$ than the case of $\psi_{0}=0^{\circ}$.

In contrast, waves with $\left|\psi_{0}\right|>20^{\circ}$ only experience a few cycles (or less) and therefore obtain smaller wave gain (generally less than $20 \mathrm{~dB}$, except for $\psi_{0}=25^{\circ}$ ). These rays are terminated due to one of the above stopping conditions. For instance, the ray with $\psi_{0}=-30^{\circ}$ leaks out of the plasmasphere (green dashed line of Figure $4 \mathrm{a}$ ) and the ray with $\psi_{0}=+30^{\circ}$ propagates to the low-altitude region (green solid line of Figure 4a) where the small wave length approximation is no longer valid. We also check the stability of cyclic raypaths for a frequency of $100 \mathrm{~Hz}$ (not shown) and find that they tend to be stable over a narrower $\psi_{0}$ range $\left(\left|\psi_{0}\right| \leq 10^{\circ}\right)$ over $15 \mathrm{~min}$, compared with $40 \mathrm{~Hz}$. 


\section{Conclusions and Discussion}

We have performed a detailed analysis of a previously reported Van Allen Probes observation of unusually low frequency plasmaspheric hiss intensification in the outer plasmasphere, using ray tracing and path-integrated wave gain calculation with inputs from in situ observations. We demonstrate that these emissions can be explained by cumulative amplification due to cyclic type of raypaths, which are caused by the sharp plasma density gradient associated with the plasmapause. Our principal conclusions are summarized as follows:

1. Plasmaspheric hiss below a few hundreds $\mathrm{Hz}$, especially at several tens $\mathrm{Hz}$, is only weakly unstable due to injected energetic electrons observed in the outer plasmasphere.

2. The cyclic raypaths are found for plasmaspheric hiss below $500 \mathrm{~Hz}$.

3. The repeated amplification over a few cycles above $60 \mathrm{~Hz}$ is capable of providing sufficient wave gain (> $40 \mathrm{~dB})$.

4. The cyclic raypaths of waves with frequency down to several tens $\mathrm{Hz}$ are relatively stable and for a range of initial wave normal angles $\left(\left|\psi_{0}\right| \leq 20^{\circ}\right)$, therefore enabling considerable amplification over a time scale comparable to a substorm injection.

There is insufficient net wave gain due to the observed electron distribution for wave frequencies between 20 and $30 \mathrm{~Hz}$, although plasmaspheric hiss at such low frequencies was observed in a spatially narrow region near $L \sim 5$. This might be due to the fact that the growth rate for such low frequencies is very weak and the fact that electron pitch angle distributions measured by the MagElS instrument were averaged over the spin period (11 s) and have limited resolution in energy and pitch angle. Therefore, the calculated growth rates using the measured electron distributions are not sufficient to counteract the Landau damping occurring during oblique propagation off the equator.

It is worth mentioning that the repeated amplification due to cyclic raypaths from a prescribed starting $L$ shell results in an apparent minimum plasmaspheric hiss intensity within the region enclosed by the raypaths. Observationally, however, no clear evidence of such minima has been reported. There are a few possibilities for the absence of the observed region with such minimum. First, the observation of the minima requires an in situ measurement by a satellite moving radially within a magnetic meridian plane. Second, spatial spreading of raypaths due to instability over a range of $L$ shells [Thorne et al., 1979] and over a range of wave normal angles (as shown in Figure 4a) might fill the outer plasmasphere and make the minimum intensity less distinct. Third, the azimuthal propagation characteristics [Chen et al., 2009] of plasmaspheric hiss waves might fill in the region of minimum wave intensity from other magnetic local times.

A follow-up study by Ni et al. [2014] has shown the effect of the unusually low frequency hiss during this event on the dynamics of radiation belt electrons. Compared with typical hiss emission, this unusual hiss even with a relatively short duration of a couple of hours, associated with substorm injection, is capable of speeding up scattering loss of electrons of energy from $50 \mathrm{keV}$ to several hundreds keV. Understanding the favorable conditions of plasmaspheric hiss emissions below $100 \mathrm{~Hz}$ is important for modeling radiation belt electron dynamics, especially during unusual periods of intense substorm injection like the event studied. This question is left as future work. Repeated amplification due to cyclic raypaths can account for the low-frequency hiss $(<200 \mathrm{~Hz})$ and be also effective for the higher-frequency hiss $(>200 \mathrm{~Hz})$. Although we do not have simultaneous observations of embryonic chorus source emissions for this particular event, previous simulations [e.g., Chen et al., 2012a] show that chorus is mainly responsible for hiss above $200 \mathrm{~Hz}$. Therefore, we suggest that hiss above $200 \mathrm{~Hz}$ might be due to a mixture of both mechanisms. The conditions favoring one or another need further investigation. Finally, it is interesting to note an observation recently reported by Tsurutani et al. [2014] that large-amplitude compressional magnetosonic waves and weak transverse electromagnetic emissions (likely hiss) were observed simultaneously by the Polar satellite, leading the authors to suggest that magnetosonic waves might contribute additionally to the formation of hiss emission.

\section{References}

Blake, J. B., et al. (2013), The Magnetic Electron Ion Spectrometer (MagEIS) Instruments Aboard the Radiation Belt Storm Probes (RBSP) Spacecraft, Space Sci. Rev., 179, 383-421, doi:10.1007/s11214-013-9991-8.

Bortnik, J., R. M. Thorne, and N. P. Meredith (2008), The unexpected origin of plasmaspheric hiss from discrete chorus emissions, Nature, 452, 62-66, doi:10.1038/nature06741. 
Bortnik, J., W. Li, R. M. Thorne, V. Angelopoulos, C. Cully, J. Bonnell, O. Le Contel, and A. Roux (2009), An Observation linking the origin of plasmaspheric hiss to discrete chorus emissions, Science, 324, 775-778, doi:10.1126/science.1171273.

Bortnik, J., L. Chen, W. Li, R. M. Thorne, and R. B. Horne (2011), Modeling the evolution of chorus waves into plasmaspheric hiss, J. Geophys. Res., 116, A08221, doi:10.1029/2011JA016499.

Chen, L., J. Bortnik, R. M. Thorne, R. B. Horne, and V. K. Jordanova (2009), Three-dimensional ray tracing of VLF waves in a magnetospheric environment containing a plasmaspheric plume, Geophys. Res. Lett., 36, L22101, doi:10.1029/2009GL040451.

Chen, L., J. Bortnik, R. M. Thorne, W. Li, and R. B. Horne (2012a), Modeling the properties of plasmaspheric hiss: 1. Dependence on chorus wave emission, J. Geophys. Res., 117, A05201, doi:10.1029/2011JA017201.

Chen, L., W. Li, J. Bortnik, and R. M. Thorne (2012b), Amplification of whistler-mode hiss inside the plasmasphere, Geophys. Res. Lett., 39, L08111, doi:10.1029/2012GL051488.

Church, S. R., and R. M. Thorne (1983), On the origin of plasmaspheric hiss-Raypath integrated amplification, J. Geophys. Res., 88, 7941-7957, doi:10.1029/JA088iA10p07941.

Draganov, A. B., U. S. Inan, V. S. Sonwalkar, and T. F. Bell (1992), Magnetospherically reflected whistlers as a source of plasmaspheric hiss, Geophys. Res. Lett., 19, 233-236, doi:10.1029/91GL03167.

Green, J. L., S. Boardsen, L. Garcia, W. W. L. Taylor, S. F. Fung, and B. W. Reinisch (2005), On the origin of whistler mode radiation in the plasmasphere, J. Geophys. Res., 110, A03201, doi:10.1029/2004JA010495.

Hayakawa, M., and S. S. Sazhin (1992), Mid-latitude and plasmaspheric HISS-A review, Planet. Space Sci., 40, 1325-1338, doi:10.1016/0032-0633(92)90089-7.

Horne, R. B. (1989), Path-integrated growth of electrostatic waves: The generation of terrestrial myriametric radiation, J. Geophys. Res., 94(A7), 8895-8909.

Huang, C. Y., C. K. Goertz, and R. R. Anderson (1983), A theoretial study of plasmaspheric hiss generation, J. Geophys. Res., 88, 7927-7940, doi:10.1029/JA088iA10p07927.

Kimura, I. (1966), Effects of ions on whistler-mode ray tracing, Radio Sci., 1, 269-283.

Kletzing, C. A., et al. (2013), The Electric and Magnetic Field Instrument Suite and Integrated Science (EMFISIS) on RBSP, Space Sci. Rev., 179, 127-181, doi:10.1007/s11214-013-9993-6.

Li, W., et al. (2013), An unusual enhancement of low-frequency plasmaspheric hiss in the outer plasmasphere associated with substorm-injected electrons, Geophys. Res. Lett., 40, 3798-3803, doi:10.1002/grl.50787.

Mauk, B. H., N. J. Fox, S. G. Kanekal, R. L. Kessel, D. G. Sibeck, and A. Ukhorskiy (2012), Science objectives and rationale for the Radiation Belt Storm Probes mission, Space Sci. Rev., 179, 3-27, doi:10.1007/s11214-012-9908-y.

Meredith, N. P., R. B. Horne, R. M. Thorne, D. Summers, and R. R. Anderson (2004), Substorm dependence of plasmaspheric hiss, J. Geophys. Res., 109, A06209, doi:10.1029/2004JA010387.

Meredith, N. P., R. B. Horne, M. A. Clilverd, D. Horsfall, R. M. Thorne, and R. R. Anderson (2006), Origins of plasmaspheric hiss, J. Geophys. Res., 111, A09217, doi:10.1029/2006JA011707.

$\mathrm{Ni}$, B., et al. (2014), Resonant scattering of energetic electrons by unusual low-frequency hiss, Geophys. Res. Lett., 40, 3798-3803, doi:10.1002/grl.50787.

Russell, C. T., R. E. Holzer, and E. J. Smith (1969), OGO 3 observations of ELF noise in the magnetosphere. 1. Spatial extent and frequency of occurrence, J. Geophys. Res., 74, 755-777, doi:10.1029/JA074i003p00755.

Smith, E. J., A. M. A. Frandsen, B. T. Tsurutani, R. M. Thorne, and K. W. Chan (1974), Plasmaspheric hiss intensity variations during magnetic storms, J. Geophys. Res., 79, 2507-2510, doi:10.1029/JA079i016p02507.

Solomon, J., N. Cornilleau-Wehrlin, A. Korth, and G. Kremser (1988), An experimental study of ELF/VLF hiss generation in the Earth's magnetosphere, J. Geophys. Res., 93, 1839-1847, doi:10.1029/JA093iA03p01839.

Sonwalkar, V. S., and U. S. Inan (1989), Lightning as an embryonic source of VLF hiss, J. Geophys. Res., 94, 6986-6994, doi:10.1029/JA094iA06p06986.

Spence, H. E., et al. (2013), Science goals and overview of the Radiation Belt Storm Probes (RBSP) Energetic Particle, Composition, and Thermal Plasma (ECT) Suite on NASA's Van Allen Probes Mission, Space Sci. Rev., 179, 311-336, doi:10.1007/s11214-013-0007-5.

Summers, D., B. Ni, N. P. Meredith, R. B. Horne, R. M. Thorne, M. B. Moldwin, and R. R. Anderson (2008), Electron scattering by whistler-mode ELF hiss in plasmaspheric plumes, J. Geophys. Res., 113, A04219, doi:1029/2007JA012678.

Thorne, R. M., E. J. Smith, R. K. Burton, and R. E. Holzer (1973), Plasmaspheric hiss, J. Geophys. Res., 78, 1581-1596, doi:10.1029/JA078i010p01581.

Thorne, R. M., S. R. Church, and D. J. Gorney (1979), On the origin of plasmaspheric hiss-The importance of wave propagation and the plasmapause, J. Geophys. Res., 84, 5241-5247, doi:10.1029/JA084iA09p05241.

Tsurutani, B. T., E. J. Smith, and R. M. Thorne (1975), Electromagnetic hiss and relativistic electron losses in the inner zone, J. Geophys. Res., 80, 600-607, doi:10.1029/JA080i004p00600.

Tsurutani, B. T., B. J. Falkowski, O. P. Verkhoglyadova, J. S. Pickett, O. SantolíK, and G. S. Lakhina (2012), Dayside ELF electromagnetic wave survey: A Polar statistical study of chorus and hiss, J. Geophys. Res., 117, A00L12, doi:10.1029/2011JA017180.

Tsurutani, B. T., B. J. Falkowski, J. S. Pickett, O. P. Verkhoglyadova, O. Santolik, and G. S. Lakhina (2014), Extremely intense ELF magnetosonic waves: A survey of Polar observations, J. Geophys. Res. Space Physics, 119, 964-977, doi:10.1002/2013JA019284.

Xiao, F. (2001), Relativistic wave-particle interaction in magnetospheric plasmas, PhD thesis, Univ. of California, Los Angeles, Calif. 